\title{
El programa Perfiles, dinámicas y desafíos de la educación costarricense: Una propuesta para la innovación pedagógica y la producción de materiales y recursos didácticos tecnológicos en la Universidad Nacional, Costa Rica
}

\author{
The National University of Costa Rica Program Profiles, Dynamics and Challenges of the Costa \\ Rican Education: A Proposal for Pedagogical Innovation and the Production of Materials and \\ Technological Didactic Resources
}

\section{O programa Perfil, dinâmicas e desafios da educação da Costa Rica: Uma proposta de inovação pedagógica e a produção de materiais didáticos e recursos tecnológicos na Universidade Nacional, Costa Rica}

Luis Alfredo Miranda-Calderón ${ }^{1}$ Universidad Nacional Centro de Investigación y Docencia en Educación Heredia, Costa Rica alfremira@gmail.com

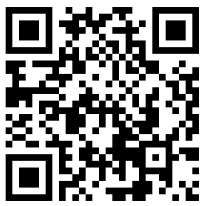

Ligia María Angulo-Hernández² Universidad Nacional Centro de Investigación y Docencia en Educación Heredia, Costa Rica ligia.maria.angulo@gmail.com

Guiselle Román-López ${ }^{3}$ Universidad Nacional Centro de Investigación y Docencia en Educación Heredia, Costa Rica guissroman@gmail.com

\footnotetext{
'Académico en la División de Educación Básica del Centro de Investigación y Docencia en Educación de la Universidad Nacional, Costa Rica y miembro del Consejo Central de Posgrado de la UNA. Doctor en Educación con énfasis en Mediación Pedagógica, Magíster con especialidades en Formación de Formadores y en Administración Educativa. Autor de diversos artículos relacionados con la capacidad creadora, la creatividad, la incorporación del juego escénico, la música y la expresión en la mediación pedagógica, así como de distintas temáticas en torno a la educación en contextos rurales. Ponente en congresos nacionales e internacionales. Actualmente es miembro del equipo académico de la carrera de Pedagogía en I y ll Ciclos y forma parte del equipo investigador del Programa Perfiles (2014 -2016), del Centro de Investigación y Docencia en Educación de la Universidad Nacional de Costa Rica.

${ }^{2}$ Académica e investigadora de la División de Educación Rural del Centro de Investigación y Docencia en Educación de la Universidad Nacional, Costa Rica. Doctora en Educación con especialidad en Mediación Pedagógica. Licenciada en Ciencias de la Educación y Máster en Docencia. Ha participado como autora de diversas publicaciones y ponencias relacionadas con temáticas de: educación y desarrollo rural, escuelas unidocentes y multigrado, investigación educativa, así como tecnología educativa. Actualmente es académica de la carrera de Licenciatura en Educación con énfasis en Educación Rural para I y II ciclos e investigadora en el Programa Perfiles (2015-2016), del Centro de Investigación y Docencia en Educación de la Universidad Nacional de Costa Rica.

${ }^{3}$ Académica e investigadora de la División de Educación para el Trabajo del Centro de Investigación y Docencia en Educación de la Universidad Nacional, Costa Rica. Magister con especialidades en Ciencias Sociales, Psicopedagogía y Estudios Latinoamericanos. Autora de diversos artículos sobre educación y diversidad, migraciones, liderazgo femenino y género. Expositora en congresos y seminarios a nivel nacional e internacional. En la actualidad se desempeña como académica en la carrera de Orientación y en la Maestría en Gestión Educativa con énfasis en Liderazgo en la División de Educación para el Trabajo y ha coordinado del Programa Perfiles (2014-2016), del Centro de Investigación y Docencia en Educación de la Universidad Nacional de Costa Rica.
} 
doi: http://dx.doi.org/10.15359/ree.22-1.10

URL: http://www.una.ac.cr/educare

CORREO: educare@una.cr

Recibido • Received • Recebido: 24 / 05 / 2016

Corregido • Revised • Revisado: 09 / 10 / 2017

Aceptado • Accepted • Aprovado: 22/ $11 / 2017$

Resumen: Se presenta la propuesta del programa Perfiles, dinámicas y desafíos de la educación costarricense, para ser ejecutada en el quinquenio 2014-2018; en el marco de los procesos de investigación, docencia, extensión y producción del Centro de Investigación y Docencia en Educación (CIDE) de la Universidad Nacional de Costa Rica. Esta propuesta plantea la implementación de dos subprogramas que abordan dos importantes áreas: la innovación pedagógica y la producción de materiales y recursos didácticos tecnológicos. El subprograma Innovación se orienta a destacar y valorar el conjunto de prácticas y experiencias exitosas en el campo de la docencia y, asume procesos vinculados a esta, tal es el caso de las actividades de práctica profesional y propuestas finales de graduación en sus distintas modalidades. El subprograma de Producción de recursos didácticos tecnológicos apoya el desarrollo de proyectos mediante la asesoría, diseño y producción de materiales y recursos didácticos tecnológicos. Se presenta la ruta metodológica que se propone para el subprograma Producción y algunos resultados preliminares logrados al inicio de la implementación de la propuesta. Finalmente se plantea una serie de reflexiones en torno a las diversas dimensiones que abarca el programa, sus alcances, impacto, desafíos y condicionantes que se deben asumir, así como las diversas aportaciones en las cuatro áreas sustantivas sobre las que se funda el quehacer de la Universidad Nacional: la docencia, la investigación, la extensión y la producción.

Palabras claves: Investigación; innovación pedagógica; producción; programas; proyectos.

Abstract: Profiles, Dynamics and Challenges of the Costa Rican Education is a program planned to be executed in a five-year period: 2014-2018. It is being executed within the framework of processes of research, teaching, university extension, and production of the Center for Research and Teaching in Education (CIDE in Spanish) of the National University of Costa Rica. This proposal considers the implementation of two sub-programs approaching two important areas: pedagogical innovation, and the production of materials and didactic technological resources. The first sub-program, Innovation, is oriented to highlighting and valuing the set of practices and successful experiences in the field of teaching, and assuming processes associated to it, such as the professional practice activities and the graduation final work proposals in different modalities. The second sub-program, Production of Didactic Technological Resources, supports the development of projects through consulting, design, and production of materials and didactic technological resources. This paper describes the methodological route proposed for the Production sub-program, along with some of the preliminary results achieved at the beginning of the implementation of the proposal. Finally, the article presents a series of reflections about the diverse dimensions that the program covers, its scope, impact, challenges, and constraints that must be assumed, as well as the different contributions in the four substantive areas on which the National University of Costa Rica bases its work: teaching, research, university extension and production.

Keywords: Research; pedagogical innovation; production; programs; projects. 
Resumo: Apresenta-se a proposta do programa Perfil, dinâmicas e desafios da educação da Costa Rica, a ser executado no quinquênio 2014-2018, no âmbito dos processos de pesquisa, ensino, extensão e produção, do Centro de Pesquisa e Ensino em Educação (CIDE), da Universidade Nacional da Costa Rica. Esta proposta sugere a implementação de dois subprogramas que abordam duas áreas importantes: a inovação pedagógica e produção de materiais e recursos didáticos tecnológicos. $\mathrm{O}$ subprograma Inovação visa destacar e avaliar o conjunto de práticas e experiências bem-sucedidas no campo da educação e assume processos relacionados a este campo, como é o caso das atividades de prática profissional e propostas finais de graduação em suas diferentes modalidades. O subprograma de Produção de recursos didáticos tecnológicos apoia o desenvolvimento de projetos através do assessoramento, projeto e produção de materiais e recursos didáticos tecnológicos. Esse trabalho apresenta o roteiro metodológico proposto para o subprograma Produção e alguns resultados preliminares alcançados no início da implementação da proposta. Finalmente, uma série de reflexões sobre as várias dimensões abrangidas pelo programa, suas limitações, impacto, desafios e condições que devem ser assumidos, bem como as diversas contribuições nas quatro áreas substantivas sobre as quais o trabalho da Universidade Nacional: ensino, pesquisa, extensão e produção.

Palavras-chave: Pesquisa; inovação pedagógica; produção; programas; projetos.

\section{Introducción}

\section{El programa Perfiles, dinámicas y desafíos de la educación costarricense}

Perfiles, dinámicas y desafíos de la educación costarricense es un programa de la Universidad Nacional que lidera procesos de investigación, docencia, extensión y producción de carácter interdisciplinario eintegrados, a cargo de un equipo compuesto por personal académico-investigador y estudiantes de las distintas unidades del Centro de Investigación y Docencia en Educación (CIDE).

En su visión, el programa propone ser el referente, a nivel de Centro e Institución, de investigación en las dinámicas y desafíos de la educación, promoviendo, así, el desarrollo y fortalecimiento del quehacer del CIDE en las líneas de innovación pedagógica, gestión y liderazgo educativo, así como en la producción de recursos didácticos-tecnológicos mediante la articulación estratégica de sus unidades académicas.

Como misión, se pretende desarrollar una serie de procesos de investigación en los ámbitos educativos y pedagógicos mediante el trabajo en equipo para generar insumos que fortalezcan y desarrollen la extensión, docencia y producción en el quehacer de las unidades académicas del CIDE, generando espacios de reflexión-acción-reflexión que contribuyan con el mejoramiento de la educación costarricense.

Mediante la implementación del programa Perfiles se pretende desarrollar una serie de procesos de investigación, que contribuyan a la construcción de conocimiento y a la generación de iniciativas innovadoras que coadyuven a fundamentar y fomentar las líneas de innovación pedagógica, investigación y producción de recursos y materiales didácticos-tecnológicos. 
doi: http://dx.doi.org/10.15359/ree.22-1.10

URL: http://www.una.ac.cr/educare

CORREO: educare@una.cr

Con la ejecución de las acciones propuestas por el programa Perfiles, además se cumple la misión histórica de la Universidad Nacional de Costa Rica que se fundamenta en su compromiso con la investigación sistemática de la realidad costarricense, en este caso, desde la formación de personal formador. Se pretende, además, generar nuevos conocimientos y contribuir al análisis ytransformación de los perfiles, dinámicas y desafíos presentes en los procesos educativos costarricenses.

Durante las últimas décadas, el Centro de Investigación y Docencia en Educación ha venido experimentando procesos de desarrollo y fortalecimiento del trabajo en las áreas de investigación, docencia, extensión y producción académica, dentro de los cuales se busca dar respuesta, tanto a las necesidades institucionales, como a las demandas y expectativas que emanan desde los procesos educativos nacionales.

A partir del año 2001, el CIDE inicia la construcción de un proyecto que contribuyera al fortalecimiento de los procesos de investigación educativa, no solo en el ámbito institucional, sino además con proyección nacional. Es para el año 2003 cuando se consolida el proyecto que recibió como nombre: Perfiles, dinámicas y desafíos de la educación costarricense e inicia su primera etapa. De esta forma, en 2004, el proyecto Perfiles, dinámicas y desafíos de la educación costarricense realizó un análisis de la situación de la educación formal en Costa Rica, lo que permitió el reconocimiento de sus principales dinámicas en los niveles de preescolar I, II y III ciclos de la Educación General Básica y Diversificada.

En su segunda etapa, a partir del 2006, se enfocó en identificar factores de éxito que han contribuido en la transición entre sexto grado de la educación primaria y sétimo año, así como en la permanencia en el tercer ciclo de Educación General Básica. Estratégicamente, se toma la iniciativa de abordar el trabajo investigativo del proyecto a partir del análisis de un conjunto de temas-problemas identificados por el Proyecto Estado de la Educación.

Uno de los temas problema que se consideró relevante considerar, por su incidencia en el campo educativo a nivel nacional, fue la deserción de estudiantes en el sétimo año de la Enseñanza General Básica o primer año de la Educación Secundaría, lo que implicó identificar los diversos factores que intervienen en esta situación. Con estos insumos y en una tercera etapa, durante 2007 se construyó el perfil de docentes de sétimo año, de manera que se logró identificar aquellos atributos, características, cualidades y condicionantes del desempeño, así como factores claves que propician una relación interpersonal positiva entre docentes y estudiantes.

En 2009, se continúa con una serie de acciones orientadas al mejoramiento de la Educación Secundaria, al construirse el perfil de docente para Ill ciclo de la Educación Pública Costarricense, según los criterios de la población de estudiantes, docentes y administrativos.

Durante el año 2010 y a partir de una serie de reflexiones del equipo investigador en torno a la coyunta educativa nacional, surge la iniciativa del Consejo Académico del CIDE de proponer que Perfiles se abocara a la ejecución de procesos de investigación en el área de las tecnologías 
de la información y la comunicación (TIC) y su integración en los procesos de enseñanza y de aprendizaje. Esta premisa consideró el desarrollo de acciones de investigación en diferentes líneas, según las diversas áreas de formación y desde las diferentes unidades académicas que conforman el CIDE, e implicó un trabajo sistemático y sostenido con diversas poblaciones de docentes y estudiantes de los diversos niveles de la Educación General Básica.

En los años subsiguientes, el proyecto logró establecer un fuerte enlace con el Ministerio de Educación Pública (MEP), lo cual generó la conformación de un equipo interdisciplinario de investigación del Centro de Investigación y Docencia en Educación y el Ministerio de Educación Pública. Este equipo desarrolló un profundo análisis descriptivo, logró identificar y analizar experiencias muy valiosas, producto del trabajo de docentes de diversas disciplinas, niveles e instituciones, en 14 regiones educativas del país.

Hoy, los resultados de todos estos esfuerzos constituyen valiosos insumos, no solo para realimentar los procesos de investigación y formación, sino además para establecer nuevas líneas de acción que garanticen la pertinencia de las futuras propuestas de investigación y subprogramas del Centro.

\section{La propuesta de Perfiles como programa para la innovación y producción en el CIDE}

A partir de 2104 y con el ideal de llevar a cabo procesos de investigación con mayor permanencia en el tiempo, así como brindar pertinencia al desarrollo de procesos de investigación, el proyecto se consolida en un programa orientado a la construcción de conocimiento y a la generación de iniciativas innovadoras, mediante la formulación de dos subprogramas que coadyuven a fundamentar y fomentar líneas de investigación en torno a la innovación pedagógica y la producción de materiales didácticos-tecnológicos.

El programa Perfiles desarrollará dos áreas de ejecución: el área de innovación pedagógica y el área de producción de materiales y recursos didácticos tecnológicos (ver Figura 1).

El área de innovación pedagógica propone la implementación de una serie de procesos articulados que implican la participación y vinculación de distintos equipos del Centro que atienden la docencia, investigación, producción y práctica profesional de las distintas carreras.

El área de producción de recursos y materiales didáctico-tecnológicos plantea dos subáreas para su ejecución. Por un lado, la asesoría y acompañamiento pedagógico, el diseño y la producción de materiales y recursos didácticos tecnológicos que desde Perfiles se les brindará a los proyectos del CIDE, con el propósito de brindar apoyo y potenciar su ejecución. Por otra parte, estos productos generados de los proyectos, así como los que se desprendan de la investigación derivada del programa innovación pedagógica ofrecerán insumos para la producción de nuevos recursos y materiales didáctico-tecnológicos, que puedan ser empleados y aprovechados en los procesos de mediación pedagógica que se gestan en las distintas unidades del CIDE. 
doi: http://dx.doi.org/10.15359/ree.22-1.10

URL: http://www.una.ac.cr/educare

CORREO: educare@una.cr

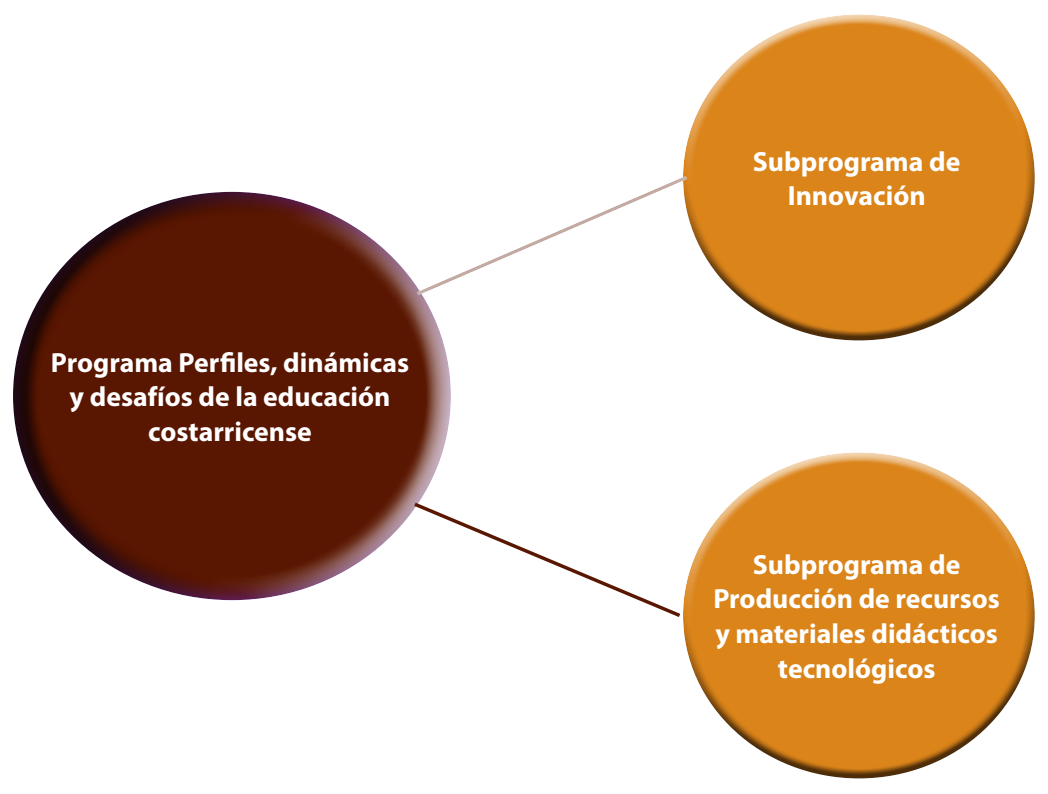

Figura 1: La propuesta del programa Perfiles implica la implementación del subprograma Innovación y el subprograma Producción de recursos.

Nota: Elaboración propia a partir de la sistematización de la propuesta.

\section{Subprograma Innovación pedagógica}

El subprograma Innovación pedagógica abordará experiencias innovadoras y exitosas en el campo de la pedagogía y la formación de personal formador que se gesta en el Centro, entendida la pedagogía como la ciencia de la educación cuyos paradigmas se vean plasmados en los distintos planes de estudio de las diferentes carreras que imparten las distintas unidades académicas del CIDE. En este caso, la innovación pedagógica debe visualizarse como la introducción de un cambio en un determinado contexto o situación, y que se da a través de una estrategia a corto plazo, con el fin de producir beneficios a través de acciones o actividades intencionales.

Como punto de partida, la innovación debe concebirse como un proceso planeado, deliberado y sistematizado, que incorpora o modifica uno o varios elementos para producir un estado de mejoría que, por ende, supone la presencia de un cambio positivo. En este caso constituye un cambio, una nueva visión del quehacer pedagógico y en la práctica profesional que no necesariamente debe caracterizar a todo el Centro, pero podría constituirse en un valioso aporte a futuro que inspire una renovada filosofía del CIDE y, consecuentemente, trascender a la comunidad educativa. 
En este contexto, la innovación se debe visualizar no solo como una estrategia pedagógica, sino además como herramienta potenciadora en los procesos de formación, que puede ser aplicada en cualquier contexto, en la práctica pedagógica de gran cantidad de docentes y en el ámbito de experiencias educativas situadas en diferentes escenarios y con distintos agentes sociales y escolares; lo que hace posible focalizarla tanto en el nivel micro, de aula, de comunidad o en el nivel macro.

\section{Objetivos del subprograma}

Los objetivos que propone el subprograma Innovación abarcan intencionalidades que involucran diversos componentes del quehacer académico del Centro; tal es el caso de la docencia, la investigación, los procesos de práctica y los aportes que se generan desde las diversas modalidades de trabajos finales de graduación que se gestan en las instancias formadoras del CIDE.

Algunos de estos propósitos se citan a continuación:

a) Fomentar la participación de docentes y del estudiantado en forma individual y comunitaria para maximizar el desarrollo de propuestas de formación con carácter innovador.

b) Identificar aquellas prácticas pedagógicas que, dadas sus características innovadoras, se orientan a la generación de cambios y mejoras en los procesos de formación y aprendizaje del Centro.

c) Destacar los modelos de innovación del Centro implementados en la formación de personal formador.

d) Determinar las condiciones del contexto específico de unidad académica y del Centro que facilitan la generación prácticas exitosas en la mediación pedagógica.

e) Reconocer, analizar y ponderar los resultados, producto de los procesos de formación e innovaciones educativas que se gestan en el CIDE.

f) Evaluar y criticar constructivamente las acciones o iniciativas de innovación de formación en el Centro con el fin de sistematizarlas para generar transformación y creación de conocimiento.

Así, se busca fortalecer el quehacer del CIDE y el desarrollo de acciones estratégicas puntuales que aporten en el impulso, acompañamiento y contribuyan a la consolidación de la política de la educación costarricense, desde la formación de personal formador y enmarcado en un contexto que propicia la innovación. 
doi: http://dx.doi.org/10.15359/ree.22-1.10

URL: http://www.una.ac.cr/educare

CORREO: educare@una.cr

El subprograma de innovación se nutrirá y se implementará a partir de la vasta gama de experiencias exitosas desarrolladas en las diferentes carreras que imparten las distintas unidades académicas del CIDE relacionadas con:

- Procesos de mediación en el campo de la pedagogía y la formación de personal formador.

- Actividades y modalidades que integran procesos innovadores, en el marco de la práctica profesional de cada una de las carreras que se imparten en el Centro.

- Trabajos finales de graduación

\section{Subprograma Producción de recursos didácticos tecnológicos}

El subprograma Producción de recursos y materiales didáctico-tecnológicos propone que, a partir del trabajo multidisciplinario, se logre concretar esta nueva dimensión de la producción académica, mediante el diseño y creación de materiales y recursos didácticos como herramientas de apoyo más acordes con el dinamismo que debe caracterizar los procesos de investigación. Del mismo modo se debe considerar la visión e intencionalidad pedagógica de cada uno de estos recursos para que, efectivamente, contribuyan a la pertinencia de los distintos procesos que se gestan desde los proyectos del Centro y su articulación con procesos de formación o las propuestas para trabajo finales de graduación.

\section{Objetivos del subprograma}

En su mayoría, los objetivos del subprograma apuntan a la concreción de una serie de pasos o etapas orientadas a la generación de recursos didácticos, principalmente en formato digital, para apoyar los proyectos que se desarrollan en el Centro. Por supuesto que, para cada uno de estos productos, su diseño debe obedecer a características muy particulares en su especificidad y de acuerdo con el propósito o intencionalidad del recurso dentro del proyecto en sí. Estos deben caracterizarse por poseer un alto potencial didáctico o una intencionalidad pedagógica, divulgativa o para la promoción de productos y resultados de cada uno de los proyectos. Entre ellos se citan los siguientes:

a) Desplegar un conjunto de acciones orientadas al fortalecimiento de los proyectos que desarrollan las distintas unidades académicas del CIDE mediante el diseño y producción de materiales y recursos didácticos tecnológicos.

b) Conformar equipos interdisciplinarios de personal académico conformados por proyectistas del CIDE y personal investigador del programa Perfiles, para la producción conjunta de materiales y recursos didácticos tecnológicos. 
c) Fomentar la participación de personal académico y estudiantes participantes en proyectos, en forma individual y comunitaria para la generación de recursos.

d) Apoyar procesos de investigación, extensión y docencia mediante la asesoría, el diseño y la producción de materiales y recursos digitales tecnológicos que enriquezcan el desarrollo de procesos de extensión y capacitación.

e) Conformar un banco de materiales y recursos digitales tecnológicos que apoyen el desarrollo de diversos proyectos del CIDE y actividades académicas vinculadas a estos.

f) Evaluar y criticar constructivamente las acciones o iniciativas de producción en el CIDE con el fin de sistematizarlas y generar la creación de conocimiento.

Para lograr estos propósitos priva la necesidad de conjuntar diversas miradas, desde las especialidades y experticia de cada una de las personas integrantes del equipo ejecutor del subprograma, sobre el ámbito de acción y sobre el objeto de estudio, en este caso, la producción de recursos. Esto, sin olvidar la complejidad y la relevancia de este tipo de actividades, si se considera que los logros y alcances de la labor que nos aboca dependen, en gran medida, de las estrategias y la forma cómo el equipo ejecutor logra aplicar sus conocimientos y complementar su desempeño mediante la integración y construcción de nuevos saberes.

Estas miradas no deben concebirse como espacios o instancias aisladas, sino que se procura que, a partir del trabajo interdisciplinario del equipo, garantizar la participación de todas y todos los sujetos involucrados en el proceso desde su especialidad y especificidad. Para que esto ocurra, se hace necesario la participación y la interacción de sus participantes en cada caso; proyectistas y personal ejecutor del subprograma. Esto garantizaría que los objetivos propuestos en el subprograma sean compartidos y viables, que las propuestas que se desarrollen estén planificadas y cuenten con un diseño adecuado a las necesidades de ambas partes, y que sean estas, necesariamente, flexibles y en beneficio tanto del subprograma como del proyecto asistido.

Una de las intencionalidades del subprograma constituye una labor de sistematización que destaque el potencial y el impacto de cada proyecto en su contexto y que describa la exploración, el recorrido y los hallazgos en torno a la creación de materiales didácticos tecnológicos, así como la construcción de nuevo conocimiento que contribuya y fortalezca la ejecución a futuro, de otros proyectos e iniciativas. Además, se propone que el diseño y producción de dichos recursos resulte adecuado y pertinente a cada propuesta, donde su uso y aprovechamiento no sea simplemente una habilidad mecánica, sino que cree un entendimiento compartido de los conceptos básicos referentes a aspectos procedimentales que privilegien la equidad e inclusión. Por tanto, el subprograma conformado por un equipo multidisciplinario de investigación iniciará en esta primera etapa del trabajo, un proceso de exploración y experimentación práctica (científica y sistemática), a partir de la visión de personal pedagogo 
doi: http://dx.doi.org/10.15359/ree.22-1.10

URL: http://www.una.ac.cr/educare

CORREO: educare@una.cr

y diseñador que le apuesta a las experiencias concretas de producir materiales y recursos que serían utilizados como soporte o apoyo de unas actividades educativas específicas, tanto para el proyecto como para la formación de los grupos formadores.

Cada subprograma descrito en esta propuesta procura ser la integración de un grupo de especialistas que conciben la investigación, la docencia, la extensión y la producción como procesos complejos; pero integrados, de construcción colectiva, donde la creación de conocimiento, la formación académica, el intercambio de saberes y la experiencia profesional orienten la generación de nuevos saberes, conocimientos y recursos contextualizados e inclusivos para atender y fortalecer la docencia, las iniciativas de formación y extensión que se desarrollan en las unidades académicas del Centro.

Se propone un espacio o punto de encuentro para la socialización de aquellas experiencias de formación que han puesto en práctica diversos enfoques en los procesos de mediación, en la metodología, en las temáticas, en la organización del aprendizaje, en el tratamiento de los contenidos, en la participación de la comunidad educativa involucrada y en la incorporación y uso de las herramientas tecnológicas como elementos potenciadores de estos procesos.

\section{Sustentación}

\section{Innovación pedagógica}

Para comprender el concepto de innovación pedagógica, es importante dilucidar el término innovación desde varias aristas que evidencien sus dimensiones.

En primer lugar, Libedinsky (2001) explica que el término innovación está compuesto por tres elementos léxicos in-, nova y -ción. In- se refiere a la introducción o ingreso de algo, Nova alude entre otros términos a nuevo, renovación, cambio y ción es un sufijo que apunta a actividad, proceso, resultado o efecto. Lo cual nos muestra de una manera clara y sencilla que se está refiriendo a introducir un cambio, algo nuevo o una renovación que implica un proceso, actividad o acción.

Castillo (2008) muestra una conceptualización que de igual manera entiende la innovación como el acto de introducir novedades, descubrir, explorar, inventar, reformar o renovar. Explica que se trata de un producto tangible que se presenta en forma intencionada, dirigido a producir beneficios y cuyos resultados son reconocidos como tales al definir la innovación.

Una estrategia a corto plazo con el fin de maximizar el beneficio o vencer una crisis inmediata como también puede ser una estrategia de mercado a largo plazo (Hannan y Silver, 2006), por lo que la innovación cobra relevancia dentro de los procesos de desarrollo dentro de las organizaciones. (p. 32) 
En la misma línea, este autor indica:

Cuando Morin y Seurat (1998) definen innovación como "el arte de aplicar, en condiciones nuevas, en un contexto concreto y con un objetivo preciso, las ciencias, las técnicas, etc. ...", están considerando que la innovación no es solamente el fruto de la investigación, sino también de la asimilación por parte de la organización de una tecnología desarrollada, dominada y aplicada eventualmente a otros campos de actividad, pero cuya puesta en práctica en su contexto organizativo, cultural, técnico o comercial constituye una novedad. (Salinas, 2004, p. 3)

De acuerdo con esto, podemos inferir cómo la innovación se puede visualizar como la introducción de un cambio en un determinado contexto o situación, y que se da a través de una estrategia a corto plazo con el fin de producir beneficios a través de acciones o actividades intencionales. En este caso en el particular, nos abocaremos al campo de la pedagogía, entendida como la ciencia de la educación, la cual es plasmada por docentes u otros agentes educativos (estudiantes, personal administrador, padres de familia, entre otros) en entornos formales o informales, y cuyo fin es que el estudiantado logre insertarse, de manera autónoma, en la sociedad basándose en el descubrimiento, análisis y reflexión de sus propias debilidades y fortalezas, según se indica en la reseña del Libro Pedagogía de la autonomía. Saberes necesarios para la práctica educativa por Freire (Paz e Terra, 2004):

Freire nos convoca a pensar acerca de lo que los maestros deben saber, y de lo que deben hacer, en el proceso de la enseñanza y el aprendizaje, sobre todo cuando el énfasis está puesto en educar para lograr la igualdad, la transformación y la inclusión de todos los individuos en la sociedad... para él la educación y las posibilidades que ella brinda de mejoramiento de la humanidad son fundamentales en su concepción sobre la liberación de los individuos y su inclusión en las sociedades. (p. 1)

Para hablar de innovación pedagógica, no basta con aplicar esta conceptualización a contextos educativos, pues, aunque esta sea una guía, se requiere de una rigurosidad mayor al utilizar el término, ya que este definiría los alcances de lo que se considera innovación pedagógica. En este sentido, (López 2011) afirma que "al momento de hacer una reflexión y una propuesta pedagógica, se hace necesario realizar una lectura juiciosa del contexto en el que se encuentra ubicado un proceso educativo. ... La pedagogía es un ejercicio político para la transformación de la [sociedad]" (pp. 17-18). 
doi: http://dx.doi.org/10.15359/ree.22-1.10

URL: http://www.una.ac.cr/educare

CORREO: educare@una.cr

Al respecto, Orterga et al. (2007) plantean:

[Una innovación educativa] puede referir a una idea, un material, una práctica, un contenido, alguna metodología, un patrón cultural, una relación entre las personas o instancias que participan en el hecho educativo, la forma de aplicar una norma, un procedimiento administrativo, un artefacto organizacional o una creencia o valor, sin ánimo de ser exhaustivos, pues hay una gran diversidad de experiencias que pueden ostentar el título de innovación educativa. (p.150)

Ante esta diversidad, Ortega et al. (2007) explican que para que se dé una innovación pedagógica se debe presentar un cambio que genere mejora en alguno de los aspectos ya mencionados, suscitando una situación o transformación original, que será novedosa hasta que sea interiorizada y se convierta en una situación normal o común. Para este equipo, las innovaciones pedagógicas dependen de factores como el contexto, los patrones culturales, el campo de conocimiento y la visión educativa en la que se enmarcan, por lo que coinciden en que no se puede hablar de un único modelo de innovación, sino que estos dependen de una cultura y un contexto determinados, e indican cómo"...el contexto es tan determinante que lo que en uno puede ser innovador, en otro, no lo es (Ortega et al., 2007, p. 150).

La innovación pedagógica involucra diferentes elementos que serán la base para los agentes participantes; esta es flexible y adaptable según el contexto en el cual será utilizada, y tendrá un límite de tiempo antes de que deje de ser considerada como innovación, pase a ser parte del ambiente en el cual nació. Es por eso que los agentes educativos que participen en su creación deben estar en constante reflexión y análisis de su práctica.

\section{Producción de materiales didáctico-tecnológicos}

Comprender el concepto de "materiales didáctico-tecnológicos" implica el análisis de teorías y definiciones alrededor de dos grandes áreas: por un lado,_qué se entiende por material didáctico; y por otro, cómo comprender un recurso tecnológico con fines didácticos. En este entendido, estamos ante una reelaboración conceptual y, si se quiere, ante la formulación de un nuevo concepto.

En primer lugar, nos referiremos al concepto de material didáctico: origen, cómo se define y qué criterios debe reunir una herramienta o recurso - de cualquier índole-para ser entendido de esta manera.

Como punto de inicio, es importante recordar el origen de los materiales didácticos. Area (2006) indica que la historia de estos es casi tan antigua como la enseñanza misma. Recalca el mérito al pedagogo y teólogo checo Juan Amos Comenio (1575-1670), por la creación del primer texto con la intención de facilitar la trasmisión de conocimientos. Además, describe la

12 Luis Alfredo Miranda-Calderón, Ligia María Angulo-Hernández y Guiselle Román-López

Los artículos de la Revista Electrónica Educare del Centro de Investigación y Docencia en Educación de la Universidad Nacional, Costa Rica, se comparten bajo términos de la Licencia Creative Commons: Reconocimiento, № Comercial, Sin Obra Derivada 3.0 Costa Rica. Las autorizaciones adicionales a las aquí delimitadas se pueden obtener en el correo: educare@una.cr 
aparición de los materiales didácticos como un proceso lento que se desarrolló gradualmente desde el siglo XVI hasta el siglo XIX, donde alcanzan su plenitud con la aparición de los sistemas escolares. Este proceso es paralelo a la consolidación de las obras impresas como elementos fundamentales del saber occidental; debido a que cuando la enseñanza empieza a generalizarse, se hace necesario contar con materiales que permitan facilitar las actividades didácticas, sistematizar los conocimientos y transmitirlos de una mejor manera. "El material didáctico, en definitiva, es un componente clave y organizador de gran parte de la actividad formativa desarrollada formalmente" (Area, 2006, p. 55).

Para Morante y Vera (2012):

El material didáctico se refiere a aquellos medios y recursos que facilitan la enseñanza y el aprendizaje, dentro de un contexto educativo, estimulando la función de los sentidos para acceder de manera fácil a la adquisición de conceptos habilidades, actitudes o destrezas. (p. 32)

Para Morante y Vera (2012) es fundamental considerar tres principios que deben ser tomados en cuenta para decidir si un recurso es o no un material didáctico. Estos principios se expresan en las siguientes condiciones indispensables que deben reunir cada obra o recurso:

La obra debe ser ... de fácil entendimiento para el público al que va dirigida.

La obra ... debe ser coherente en todas sus partes y en todo su desarrollo vistoso.

La obra ... debe contener los recursos suficientes para que se puedan verificar y ejercitar los conocimientos adquiridos por el estudiante. (p. 32)

Por su parte Cacheiro (2011, citando a Blázquez y Lucero, 2002) señalaba como recurso didáctico:

[cualquiera que] el profesor prevea emplear en el diseño o desarrollo del currículo ... para aproximar o facilitar contenidos, mediar en las experiencias de aprendizaje, provocar encuentros o situaciones, desarrollar habilidades cognitivas, apoyar ... estrategias metodológicas, ... facilitar o enriquecer la evaluación. (p. 70)

Para este autor, es necesario retomar las funciones que los medios didácticos cumplen. En este caso, Cacheiro (2011) se vale de Márquez (2000), quien expone como fundamentales seis aplicaciones del material didáctico: 
doi: http://dx.doi.org/10.15359/ree.22-1.10

URL: http://www.una.ac.cr/educare

CORREO: educare@una.cr

1. Motivar, despertar y mantener el interés;

2. Proporcionar información;

3. Guiar los aprendizajes de los estudiantes: organizar la información, relacionar conocimientos, crear nuevos conocimientos y aplicarlos...;

4. Evaluar conocimientos y habilidades;

5. Proporcionar simulaciones que ofrecen entornos para la observación, exploración y la experimentación;

6. Proporcionar entornos para la expresión y creación. (p. 71)

En este sentido es destacable el aporte de Ruz (2010), quien señala como vitales las fases de diseño y elaboración de materiales. Esta autora propone tres fases de diseño que se cruzan y difuminan entre sí. Inicialmente, Ruz (2010) se refiere a una fase de preproducción, en la que se recuperan datos y se evalúan, de forma participativa, las necesidades o demandas del contexto o la población que emplearía el material. Luego, viene una fase de producción, donde, retomando las sugerencias y hallazgos de la fase anterior, se delimitan objetivos, contenidos, soporte, equipo requerido y presupuesto. Por último, la fase de posproducción valida el material construido, evaluando su utilidad, su claridad, el cumplimiento de objetivos, etcétera; y con base en los resultados de esta valoración, se hacen los últimos ajustes.

Reconstruyendo lo anterior, podemos decir que la didáctica es un ejercicio de construcción colectiva, donde los diferentes actores participan, idealmente, en un entorno de comunicación horizontal y contextualizada.

El siguiente concepto que tendríamos que definir es el de recursos tecnológicos con fines didácticos.

Area (2006) señala que, con la llegada de la imprenta y las ideas de la democratización de los grupos enciclopedistas, en el siglo XIX se impulsó un modelo escolar donde el aprendizaje se desarrollaba a través de los textos, en lo que se denomina "cultura impresa". No obstante, con el paso del tiempo esto ha cambiado, ya que la información se transmite por medio de imágenes, sonidos y la combinación de estos.

Con la llegada de las tecnologías de la información y comunicación (TIC), las formas de almacenar y difundir la información han variado, por lo que las formas de transmitir, organizar y procesar el conocimiento varían y reclaman también cambios en los modelos con los que tradicionalmente se han llevado a cabo los procesos de enseñanza y aprendizaje. 
De acuerdo con lo anterior, cobra importancia la definición de recursos tecnológicos con fines didácticos. A este respecto, Cacheiro (2011, retomando a Medina, 2009), menciona una clasificación que define por tipos lo que se consideraría un recurso tecnológico. En este documento se habla de tres clases: los recursos de información, los recursos de colaboración y los recursos de aprendizaje.

Porúltimo, los recursos TIC para el aprendizaje posibilitan la construcción de conocimientos y de actitudes formativas. Estos recursos, en concreto, permiten diversas maneras de abordar contenidos y desarrollar actividades para la apropiación o reformulación de estos. Para Cacheiro (2011), "permiten pasar de un uso informativo y colaborativo a un uso didáctico para lograr unos resultados de aprendizaje" (p. 75).

Siguiendo con lo anterior, Area (2003) explica cómo algunos de los sitios web educativos pueden ser usados como materiales didácticos, partiendo de una propuesta clasificatoria que incluye webs institucionales, webs de recursos y bases de datos, webs de teleformación y materiales didácticos en formato web. Este autor incluye los webs tutoriales, webs docentes o materiales didácticos en formato web como páginas confeccionadas para ser utilizadas en procesos de enseñanza y aprendizaje.

\section{La ruta metodológica}

El abordaje de los procesos para concretar esta propuesta implica la implementación de una serie de actividades paralelas que se proponen en cada uno de los subprogramas.

Se plantea desarrollar dos áreas clave: innovación educativa y producción de recursos y materiales didáctico-tecnológicos que, aunque son independientes entre sí, poseen puntos de encuentro y convergencia en la metodología. Tal es el caso de actividades como:

- Revisión documental

- Selección de información

- Consulta y entrevistas a personas participantes en proyectos

- Procesos de producción de recursos para los proyectos

- Procesos de sistematización de procesos (producción)

- Análisis de información

La ruta metodológica de la propuesta para ambos subprogramas implica una serie de procesos graduales y flexibles que centran sus metas en la vinculación con los diversos actores del Centro. Lo que involucra un proceso dinámico y creativo de construcción, que se nutre y 
doi: http://dx.doi.org/10.15359/ree.22-1.10

URL: http://www.una.ac.cr/educare

CORREO: educare@una.cr

se redefine sobre la marcha con las distintas etapas que se irán desarrollando. En el caso del subprograma de producción, los distintos momentos que se implementaron en la etapa inicial permitieron trazar un camino tentativo que constituye la base para la creación de un protocolo o itinerario de producción (ver figura 2).

\section{Trazando el camino}

A continuación, se describen los momentos que caracterizaron el abordaje y las experiencias del proceso de producción de recursos y materiales didáctico-tecnológicos con algunos de los proyectos que participaron en la etapa inicial.

A partir de la experiencia en la primera etapa, la secuencia que se describe a continuación pretende ser una hoja de ruta que oriente, en primera instancia, la planificación y el desarrollo de este tipo de procesos desde una visión dialógica e interdisciplinaria.

Los seis momentos detallan el paso a paso de la experiencia llevada a cabo por el equipo de Perfiles en la concreción de la producción de recursos y materiales didáctico-tecnológicos.

El trayecto se concibe como un camino flexible y en proceso de construcción, por lo que continuará siendo enriquecido en los momentos próximos de producción y de fortalecimiento de vínculos entre los proyectos, programas y actividades académicas del CIDE, según las especificidades y contextos de cada uno.

\section{Primer momento: Diagnóstico}

Posibilitó conocer y socializar, entre integrantes del equipo, el estado del arte de los proyectos formulados en las diferentes unidades académicas del CIDE.

\section{Segundo momento: Preselección}

Se llevó a cabo una sesión de análisis para seleccionar de forma preliminar los proyectos que conformarían la etapa inicial de producción, con base en los criterios de vigencia, solvencia financiera e interés en generar vínculos con Perfiles. Precisamente, por las mismas dinámicas y características de los proyectos, como los plazos de vigencia, dicha preselección fue de carácter tentativo.

\section{Tercer momento: Contacto con proyectistas seleccionados}

Una vezescogidos los proyectos, se procedióa realizar encuentros individualescon distintas modalidades (entrevistas semiestructuradas, reuniones formales e informales, comunicación 
por medios electrónicos, entre otros) con las personas coordinadoras de los proyectos, con el propósito de compartir información general del programa y conocer su nivel de interés para trabajar mancomunadamente con Perfiles en la producción de los materiales y recursos.

\section{Cuarto momento: Selección}

A partir de la preselección, de los vínculos establecidos y de discusiones sostenidas a lo interno del equipo Perfiles, se llevó a cabo un proceso de análisis sobre los criterios de selección iniciales que resultó en un cambio de rumbo con respecto a la forma en que se venían seleccionando los proyectos. En este sentido, se propuso extender la invitación a proyectistas que desearan participar y vincularse con el programa.

\section{Proyectos participantes para la etapa inicial}

En total se contó con ocho proyectos seleccionados. Sus plazos de vigencia van de los 3 a los 5 años, según respondan a la categoría de proyectos, programas o actividades académicas. Se espera que, con la apertura de criterios, se alcance a más proyectos.

La mayoría de los proyectos son de carácter interdisciplinario, lo que subraya la naturaleza extensiva de sus propuestas. Solo uno de ellos es multidisciplinario y tres, disciplinarios. Además, integran diversas áreas sustantivas de la Universidad Nacional, mediante distintas modalidades como lo son: docencia-investigación, docencia-investigación-extensión y docencia-investigación-extensión-producción.

Son muy pocos los que se circunscriben únicamente a actividades de extensión o investigación. Las combinaciones son afines a los planteamientos del Modelo Pedagógico de la Universidad y evidencian un esfuerzo por integrar y enriquecer el quehacer universitario.

Se dirigen a diversas poblaciones meta. Abarcan desde estudiantes y docentes de la misma unidad académica de la Universidad Nacional, hasta actores externos de otras instituciones públicas tales como la Universidad de Costa Rica, la Caja Costarricense del Seguro Social, el Ministerio de Educación Pública, instituciones educativas de primaria y secundaria, así como organismos no gubernamentales y grupos sociales específicos (personas con discapacidad, comunidades indígenas, profesionales en servicio de la educación). Además, algunos proyectos cuentan con la asistencia y colaboración de estudiantes de las carreras en educación; otros, funcionan como plataforma para articular propuestas finales de graduación con el objeto de estudio de los proyectos.

La diversidad de actores e instituciones que participan en los proyectos del CIDE constituye una pequeña muestra de las redes, dinámicas y atención extendidas de la Universidad Nacional a las distintas poblaciones de la comunidad nacional, en consistencia con el enfoque 
doi: http://dx.doi.org/10.15359/ree.22-1.10

URL: http://www.una.ac.cr/educare

CORREO: educare@una.cr

de universidad necesaria que la caracteriza. Asimismo, estas articulaciones se traducen en oportunidades para gestar experiencias orientadas a la cooperación y los inter aprendizajes.

Los objetos de estudio son diversos y apuntan hacia ámbitos fundamentales en procesos de educación formal o informal. Destacan, por ejemplo, la mediación pedagógica a través de la integración crítica de las tecnologías de la información y la comunicación, la práctica docente, los servicios y apoyos en la atención de las necesidades educativas de la población estudiantil de la UNA, la educación continua para docentes en servicio, estudiantado egresado de Pedagogía con énfasis en I y II Ciclo y profesionales afines, la pedagogía social, y el fomento, animación y promoción de la lectura. Los productos esperados son de distinta naturaleza e incluyen documentos tales como: artículos, ponencias, informes parciales y finales, planeamientos, unidades didácticas, videos y boletines, entre otros.

Las pautas de producción se construyen en conjunto desde un enfoque interdisciplinario donde se consideran tanto los aportes del equipo Perfiles como los proporcionados por proyectistas de las unidades académicas. Asimismo, los lazos entre Perfiles y los diversos proyectos se configuran a partir de las especificidades y necesidades que presenta cada caso, lo que le da un carácter abierto, dinámico, inacabado y de proceso. Esta nueva apertura responde al fuerte interés de Perfiles en consolidar la red de vínculos inter-unidades del Centro y, con ello, trascender los objetivos propuestos en la formulación oficial del programa, de tal manera que los beneficios no se limiten a la mera producción, sino que se extiendan a otros planos del quehacer del CIDE.

\section{Quinto momento: Definición del proceso para la producción de los recursos}

Como parte del trabajo en equipo de Perfiles, se generaron discusiones profundas con respecto al proceso para la producción de los recursos. De dichas discusiones surgió la necesidad de examinar una serie de elementos clave para la articulación y la definición del proceso, motivo por el cual se realizó una indagación sobre productos que podrían generarse, se revisaron diversas normativas institucionales para identificar las que rigen estos procesos y se definieron compromisos académicos, éticos y técnicos para los participantes (equipo Perfiles y proyectistas).

Sumado a lo anterior, las discusiones generaron la necesidad de ejecutar un producto con el fin de revisar y clarificar procedimientos.

Este primer ejercicio evidenció la necesidad de definir un protocolo para la producción, entrega oficial del producto y la evaluación del proceso por parte del grupo de proyectistas. Asimismo, a las pautas definidas durante este proceso, se suman encuentros grupales eindividuales con proyectistas, con el propósito de concretar las líneas de acción que se seguirán en conjunto. 
doi: http://dx.doi.org/10.15359/ree.22-1.10

\section{Un itinerario para el proceso de producción}

Como resultado de un profundo análisis que consideró las expectativas y aprendizajes durante estos seis momentos; este proceso se sistematizó a manera de itinerario y en función de la producción de los recursos para los proyectos, mediante una serie de cinco pasos o fases que integran la asesoría pedagógica y de diseño, la innovación educativa y el trabajo colaborativo; se delimitan las instancias y personas participantes, las responsabilidades y compromisos, así como la dinámica de producción de estos recursos y materiales; se inicia desde la concepción del material o recurso didáctico en lo interno de cada proyecto, hasta la aplicación y evaluación final de cada uno de estos productos (ver Figura 2).

\section{Pasos de la ruta de producción}

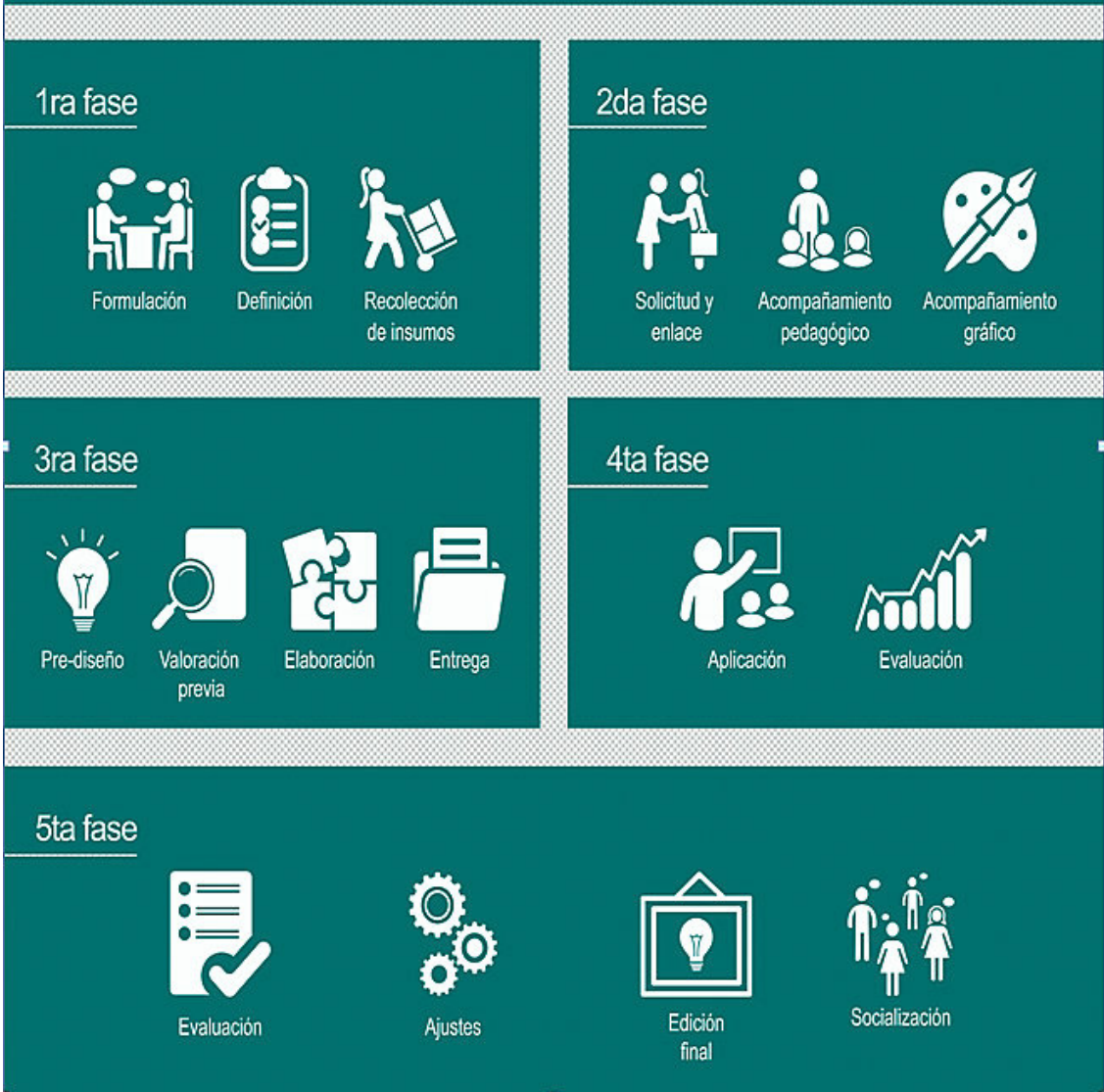

Figura 2: Pasos secuenciales de la ruta de producción de recursos didácticotecnológicos.

Nota: Elaboración propia a partir de la sistematización de la propuesta. 
doi: http://dx.doi.org/10.15359/ree.22-1.10

URL: http://www.una.ac.cr/educare

CORREO: educare@una.cr

\section{Sexto momento: En la praxis}

Corresponde a la fase actual, de contacto e interacción con proyectistas, donde se da el intercambio de ideas, la realimentación y la puesta en marcha de acciones para concretar el diseño y la producción de los recursos y materiales. Por lo tanto, se puede afirmar que el logro de los alcances de la creación de materiales didáctico-tecnológicos está intrínsecamente ligado a los niveles de interés, participación y procesos de interacción, comunicación y colaboración que se puedan gestar en el camino.

\section{Retos y desafíos}

Como en toda actividad académica que apunte al cambio, la ejecución del programa presupone afrontar una serie de desafíos que implican la adquisición de nuevas habilidades y la disponibilidad al cambio.

Con la ejecución del programa, así como la formulación y el desarrollo de los dos subprogramas, se asume el desafío que supone la conformación de un equipo ejecutor de la propuesta con perfil interdisciplinario, para apoyar e innovar en la puesta en práctica de una iniciativa que desde su génesis se caracteriza por ser innovadora.

Incursionar en el campo de la producción de recursos implica un gran reto, pero al mismo tiempo una interesante oportunidad de aprendizaje para todas las personas vinculadas al programa, ya que involucra una serie de cambios, no solo en la forma de trabajo investigativo que incorpora el componente de diseño y producción gráfica, sino además en la actitud académica con disposición al cambio y la innovación, que debe caracterizar al equipo investigador.

Otro gran reto de la propuesta es concretar la dimensión interdisciplinaria en la dinámica de trabajo integrado que se requiere para el logro de los objetivos propuestos, lo que implica la participación de académicas y académicos de las distintas unidades, carreras y proyectos, como elemento fundamental para la ejecución de la propuesta. Esta metodología propiciaría el intercambio de saberes entre las personas participantes y el mutuo enriquecimiento con temáticas de producción e innovación educativa, entre otras áreas afines al Programa que están presentes en los proyectos y las carreras del CIDE. Por tales razones, se plantea el compromiso de generar acciones académicas que traspasen los límites de cada unidad, con el propósito de conformar equipos de trabajo inter-unidades que privilegien la interacción y el intercambio de conocimientos que puedan aportar a la investigación y a la producción.

Se aspira a que los subprogramas se constituyan en estrategias de centro para la integración e interacción entre las distintas unidades académicas, con el fin de concurrir en la satisfacción de las demandas de formación de estudiantado. Por lo tanto, se pretende diagnosticar, detectar y valorar provisoriamente aquellas acciones o actividades innovadoras que promuevan la formación universitaria sólida e integral de profesionales en el campo de la pedagogía.

\footnotetext{
20 Luis Alfredo Miranda-Calderón, Ligia María Angulo-Hernández y Guiselle Román-López
} 


\section{Reflexiones y aprendizajes: Primeros logros al inicio del camino}

En este apartado se exponen algunas reflexiones generadas a partir del camino recorrido hasta el momento en el área que se viene mencionando.

El Centro de Investigación en Docencia y Educación se ha caracterizado por su interés en entender los retos actuales al promover nuevas estrategias para educar desde una visión integral e innovadora, que responde a la misión y visión de la UNA y la intención de promover en el estudiantado una formación humanística integral y conocimientos, destrezas y habilidades acordes con las necesidades de la sociedad.

El conjunto de proyectos que se gestan en el CIDE han estado liderados por un grupo de proyectistas que conciben la investigación, la docencia, la extensión y la producción como procesos complejos e integrados a la vez. Esta coyuntura le ha permitido, a este Centro, la construcción permanente y colectiva del conocimiento, la formación académica, el intercambio de saberes para atender y fortalecer la docencia, las iniciativas de formación y extensión que se desarrollan en las diferentes unidades académicas que lo conforman.

Por ende y considerando estas oportunidades del Centro, el proyecto Perfiles en su última etapa de vigencia en el año 2015 propuso la ejecución de una iniciativa de Centro que les implicó, a quienes integran el equipo, la adquisición de nuevas habilidades y disponibilidad para elaborar esta propuesta que fue considerada desde su génesis, como innovadora. La propuesta descrita anteriormente se vislumbró como una oportunidad para crear y renovar este Centro de formación y, por ende, las intencionalidades adscritas a cada uno de los proyectos que se desarrollaban en ese momento y potenciar, así, la comunicación y trabajo articulado entre el CIDE y otros espacios de la Universidad.

En el año 2015, el equipo de investigación de Perfiles lideró algunas de las acciones indicadas en la propuesta, dirigidas a la producción de recursos y material didáctico-tecnológicos por implementarse en los procesos de mediación pedagógica en la formación de nuevo personal docente. Esta iniciativa surge de un proceso de análisis interdisciplinario que refleja la concepción de los objetivos de los proyectos de las unidades académicas, en donde se muestra el interés de que estos procesos integrados fortalezcan las prácticas educativas y propicien futuros grupos docentes con una mirada crítica y actualizada de la realidad costarricense. Por lo tanto, esta tarea debe garantizar la construcción de una sucesión de actos y de acontecimientos para alcanzar metas educativas y aprendizajes comunes a todas las personas involucradas y que responda al compromiso social de Universidad Nacional en la actual coyuntura.

La propuesta considera la oportunidad de generar procesos interdisciplinarios que permitieran comprender las realidades de las poblaciones con las que se trabajó, desde una óptica integral. Sin embargo, en muchos casos, estos esfuerzos se han visto reducidos a procesos que, por lo general, se abordan únicamente al interior de cada unidad académica y los esfuerzos por comunicar y socializar los alcances y productos son limitados. 
doi: http://dx.doi.org/10.15359/ree.22-1.10

URL: http://www.una.ac.cr/educare

CORREO: educare@una.cr

Algunas de las actividades que lograron ser desarrolladas detectaron la importancia que tiene cada proyectista del Centro de asesoramiento para el diseño, la producción de recursos y materiales didácticos tecnológicos que pueden elaborar desde las acciones de cada proyecto. Además, que el diseño y producción de dichos recursos resulte adecuado y pertinente, donde su uso y aprovechamiento no sea simplemente una habilidad mecánica, sino que cree un entendimiento compartido de los conceptos básicos referentes a aspectos procedimentales que privilegien la equidad e inclusión. Estos mismos, caracterizados por poseer un alto potencial didáctico o una intencionalidad pedagógica.

Incursionar es estas dos áreas de la propuesta implicó, para sus gestores y gestoras, y para las personas vinculadas a su accionar, un gran reto, pero al mismo tiempo una oportunidad de aprendizaje de trabajo investigativo desde una visión interdisciplinaria con una metodología de intercambio de saberes entre las personas participantes

Esta experiencia le permitió al equipo de Perfiles conocer la importancia del rol de la persona profesional en diseño gráfico integrada al equipo, ya que los distintos espacios de trabajo interdisciplinario que sostuvo la diseñadora y proyectistas para la creación y producción de boletines informativos y catálogos de producción intelectual, de las unidades académicas, así como brochures, afiches e imagotipos, entre otros productos que le dan identidad propia a los proyectos, garantizaron aspectos de estética en el diseño, pertinencia y funcionabilidad, y cubrieron, así, exigencias prácticas acordes con las expectativas de cada proyecto.

Otras acciones igualmente exitosas e innovadoras resultantes de esta propuesta pedagógica, constituye el haber gestionado acciones conjuntas con participación de personal académico y estudiantes del CIDE con el Centro de Investigación, Docencia y Extensión Artística (CIDEA), específicamente con el Programa Integrado Conexiones para la Creatividad. Todos estos resultados señalados de manera ilustrativa de la implementación de algunas de las acciones suscritas a la propuesta permitieron comprobar que tanto la innovación educativa como el área de la producción de materiales y recursos didácticos - tecnológicos constituyen una necesidad en el Centro que sugieren el requerimiento de fortalecer y renovar estas áreas.

Con la puesta en práctica del subprograma de Innovación se podrían identificar diversos espacios para analizar cómo se gestan y desarrollan prácticas innovadoras y de qué forma impactan el quehacer académico y aportan al alcance de logros educativos. Además, posibilita conocer, con detalle, quién es la persona promotora, cuál es la finalidad de su aplicación y quién o quiénes son el público usuario o posibles grupos beneficiarios. De esta manera, la innovación podría concebirse como un aporte novedoso que contribuya a una mejor consecución de los objetivos de los programas de formación desarrollados en la Universidad y en el marco de la actual coyuntura sociohistórica. 
La implementación del subprograma de Innovación fomentaría, en el CIDE, el liderazgo, la iniciativa, el trabajo interdisciplinario y el potencial de todos los estilos de trabajo que desarrollan las académicas y los académicos formadores. Por otro lado, se podría dar cabida para la realización de otros proyectos, tanto de carácter técnico como didáctico.

Algunas de las ideas que se sugieren giran en torno al fomento y al desarrollo de la innovación pedagógica en la formación de las diferentes carreras que se imparten en el CIDE, en la innovación tecnológica del Centro en aspectos de profundización pedagógica, en el uso y aprovechamiento de las nuevas tecnologías en correspondencia con la actual coyuntura, o en el desarrollo de proyectos con colaboración entre unidades, personal académico y en estrecha vinculación con otros centros de formación.

Esta propuesta se vislumbra como una ventana abierta que da la oportunidad para acceder a nuevos saberes, explorar nuevas posibilidades en el campo de la pedagogía, renovar nuestro centro de formación y, por ende, las intencionalidades adscritas a cada uno de los proyectos que se desarrollan en esta instancia. Una actividad que se pretende que sea asumida a partir del trabajo colaborativo y cooperativo y que propicie, desde la visión multidisciplinaria, la interacción académica intra e inter-unidades.

Se insta a la comunidad CIDE a continuar diagnosticando, detectando y valorando provisoriamente aquellas acciones o actividades en el área de la innovación y producción de materiales que promuevan la formación universitaria sólida e integral de profesionales en el campo de la pedagogía.

\section{Referencias}

Area, M. (2003). De las webs educativas al material web. Comunicación y pedagogía: Nuevas tecnologías y recursos didácticos, 188, 32-38.

Area, M. (2006). De los libros de texto a los materiales didácticos web. En M. Á. M. GarcíaQuismondo y L. B. Andreu (Coords.), La biblioteca: Un mundo de recursos para el aprendizaje (pp. 53-82). España: Ministerio de Educación Pública, Secretaría General Técnica.

Cacheiro, M. L. (2011). Recursos educativos. TIC de información, colaboración y aprendizaje. Pixel-Bit. Revista de Medios y Educación, 39. Recuperado de http://www.redalyc.org/ articulo.oa?id=36818685007

Castillo, P. (2008). 2. Cambios, novedades y procesos de innoación. En J. Salinas (Coord.), Innovación educativa y uso de las TIC (pp. 31-42). Sevilla: Universidad Internacional de Andalucía. Recuperado de http://dspace.unia.es/bitstream/handle/10334/133/004tic castillo debenito.pdf?sequence $=1$ 
doi: http://dx.doi.org/10.15359/ree.22-1.10

URL: http://www.una.ac.cr/educare

CORREO: educare@una.cr

Libedinsky, M. (2001). La innovación en la enseñanza. Diseño y documentación de experiencias en el Aula. Mexico, D. F.: Paidós.

López, D. (2011). Simón Rodríguez y José Martí: Rasgos iniciales de pedagogía crítica latinoamericana. Ensayos Pedagógicos, 6(2). Recuperado de http://www.revistas.una.ac.cr/ index.php/ensayospedagogicos/article/view/5816/5684

Morante, L y Vera, I. (2012). Materiales didácticos tecnológicos innovadores para la enseñanza de Estudios Sociales (Tesis de licenciatura). Universidad Estatal de Milagro. Ecuador. Recuperado de http://repositorio.unemi.edu.ec/bitstream/123456789/257/3/ MATERIALES DIDACTICOS TECNOLÓGICOS INNOVADORES PARA LA ENSEÑANZA DE ESTUDIOS SOCIALES.pdf

Ortega, P., Ramírez, M. E., Torres, J. L., López, A. E., Servín, C. Y., Suárez, L. y Ruiz, B. (2007). Modelo de innovación educativa. Un marco para la formación y el desarrollo de una cultura de la innovación. RIED. Revista Iberoamericana de Educación a Distancia, 10(1), 145-173. Recuperado de http://www.redalyc.org/articulo.oa?id=331427206010

Paz e Terra. (2004). Reseña del libro Pedagogía de la autonomía. Saberes necesarios para la práctica educativa, por P. Freire. Sao Paulo: Autor.

Ruz, I. (2010). Diseño y elaboración de materiales informatizados en el ámbito de la orientación educativaylatutoría.Pixel-Bit.RevistadeMediosyEducación,36,41-51.Recuperadodehttps:// idus.us.es/xmlui/bitstream/handle/11441/22611/file 1.pdf?sequence=1\&isAllowed=y

Salinas, J. (2004). Cambios metodológicos con las TIC. Estrategias didácticas y entornos virtuales de enseñanza-aprendizaje. Bordón. Revista de Pedagogía, 56(3-4), 469-481. 